\title{
How reliable are capillary blood glucose measurements?
}

\author{
H N Rajaratnam ${ }^{1}$, S Pathmanathan ${ }^{2}$ \\ Sri Lanka Journal of Diabetes Endocrinology and Metabolism 2011; 1:22-24
}

Capillary blood tests measure whole blood glucose as opposed to venous samples which measure plasma glucose. It is used for the care of people with diabetes, as a monitoring tool, giving a guide to blood glucose levels, at a specific moment in time. Capillary blood glucose monitoring was first established in the 1970s using glucometers. With time, the use of glucometers has become easier and faster, with much smaller blood samples, yielding results in a matter of seconds. Today they are used routinely in health care, for the easier achievement of glycaemic targets and diabetic emergencies. Without such technology, intensive glucose control including insulin pump therapy would not have become a reality. Glucometers have also relieved a great amount of anxiety over the management of hypoglycaemia. Today however, we rely so much on capillary blood glucose measurements forgetting its limitations. This article will discuss the pitfalls and limitations of capillary blood glucose monitoring $(1,2)$.

\section{Accuracy goals for home glucose monitors}

The goals for glucometer accuracy have been quite variable. Clarke et al. proposed an accuracy grid to establish a more expansive set of goals for glucometer usage taking into account clinical accuracy, defined as within $20 \%$ of the laboratory glucose (3). For glucose levels above $75 \mathrm{mg} / \mathrm{dl}$, the International Organization for Standardization (ISO) recommends a goal for glucometer error of within $20 \%$ when compared with a reference glucose sample, but for glucose levels less than $75 \mathrm{mg} / \mathrm{dl}$, the goal is for $95 \%$ of readings to be within $15 \mathrm{mg} / \mathrm{dl}$ of the reference. The U.S. Food and Drug Administration goal for glucometers is within $20 \%$ of the reference value, when glucose is greater than $100 \mathrm{mg} / \mathrm{dl}$ and within $20 \mathrm{mg} / \mathrm{dl}$ when glucose is less than $100 \mathrm{mg} / \mathrm{dl}(4,5)$.

Although there is no universally binding standard, guidelines issued by ISO are widely acknowledged. Assuming a meter meets the ISO guideline, then a true glucose level of $55 \mathrm{mg} / \mathrm{dl}$ could in fact yield a reading of as low as 40 or as high as $70 \mathrm{mg} / \mathrm{dl}$. It could be particularly hazardous in a patient with hypoglycaemia unawareness who would consider the reading of $70 \mathrm{mg} / \mathrm{dl}$ as reassuring for a true value of $55 \mathrm{mg} / \mathrm{dl}$, which needs prompt corrective action. At the other end of the spectrum, a true value of
$350 \mathrm{mg} / \mathrm{dl}$ might register as low as 280 or as high as 420 $\mathrm{mg} / \mathrm{dl}$. This could have some consequences, especially in intensive care situations, where insulin infusion algorithms aim at achieving tight glycaemic control (4).

\section{Multiple variables affecting glucometer values} Preanalytical variables

A number of preanalytical variables can also potentially cause inaccuracy in glucometer measurements. Haematocrit (greater than 55\% may lead to inaccurate results when the blood glucose level exceeds $11 \mathrm{mmol} / 1$.), temperature, hypoxia, humidity, severe hypo- or hyperglycemia, peripheral circulatory failure, elevated cholesterol ( $>13 \mathrm{mmol} / \mathrm{l})$ and some drugs, such as ascorbic acid are recognized variables.

In patients with peripheral circulatory failure and severe dehydration (eg: diabetic ketoacidosis, hyperosmolar non-ketotic coma), shock and hypotension may occur. In these situations capillary blood glucose readings can be artificially low due to peripheral shut down, leading to increased glucose extraction by the tissues, because of low capillary flow and increased glucose transit time. Similarly capillary blood glucose measurements may not be reliable in patients who have defects in microcirculation, such as, those with Raynaud phenomenon and severe peripheral vascular disease (6).

\section{User error/operator error}

The technique of the user or operator of the glucometer usually is responsible for more inaccuracy than the glucometer itself. Applying insufficient blood to the strip, using strips that are out of date or exposed to excess moisture or humidity, and failure to enter the proper code, can compromise accuracy $(2,7)$.

Several important technologic advances that decrease operator error have been made in the last few years. These include "no wipe" strips, automatic commencement of timing when both the sample and the strip are in the meter, smaller sample volume requirements, an error signal if sample volume is inadequate, "lock out"

${ }^{1}$ Consultant Physician and Endocrinologist, Nawaloka Hospitals Limited,Colombo 2, ${ }^{2}$ Senior Registrar in Endocrinology, National Hospital of Sri Lanka. 
if controls are not assayed, barcode readers, and the ability to store up to several hundred results that can subsequently be downloaded for analysis. Together these improvements have produced superior performance by newer meters $(2,7)$.

\section{New technologies: alternative site testing}

Some glucometers allow testing blood from alternative sites, such as the upper arm, forearm, base of the thumb, and thigh. Sampling blood from alternative sites may be desirable, but has some limitations. Blood in the fingertips show changes in glucose levels more quickly than blood in other parts of the body. This means that alternative site test results will be different, not because of the meter's inability to test accurately, but because the actual glucose concentrations are different. The FDA believes that further research is needed to better understand these differences in test values, and their possible impact on the health of people with diabetes (8).

\section{Choosing the correct blood sample}

There are several aspects concerning the blood sample that needs attention. Although there are different recommendations, the first choice is to wash the hands with soap and water, dry them, and use the first drop of blood for assessment. Erroneous blood glucose levels (pseudo hyperglycaemia) have been recorded when patients did not wash their hands with water after peeling fruits and such false readings were still noted when hand washing was substituted with the use of an alcohol swab. If washing hands is not possible, and they are not visibly soiled or exposed to a sugar-containing product, it is acceptable to use the second drop of blood after wiping away the first drop. Firm squeezing of the finger should be avoided $(9,10)$.

\section{Analytical variables}

\section{Whole blood glucose vs. plasma glucose}

Glucose levels in plasma are generally $10-15 \%$ higher than glucose measurements in whole blood (and even more after eating). This is important because home blood glucometers measure the glucose in whole blood, while most laboratory tests measure the glucose in plasma. There are many meters on the market now that give results as "plasma equivalent". This allows patients to easily compare their glucose measurements in a lab test and at home. The meters that give "plasma equivalent" readings have a built in algorithm, that translates the whole blood measurement, to make it seem like the result that would be obtained on a plasma sample. It is important to know whether the particular meter gives its results as "whole blood equivalent" or "plasma equivalent" $(8,9,10)$.

\section{Enzymatic measurement of glucose concentration}

Clinical laboratories estimate glucose concentration based on enzymatic measurement of hexokinase which is the gold standard, while in test strip systems, glucose concentration is based on enzymatic measurement of glucose oxidase, glucose dehydrogenase nicotinamide adenine dinucleotide (GDH-NAD), GDH flavin adenine dinucleotide (GDH-FAD) and GDH pyrroloquinolinequinone (GDH-PQQ). Sensors based on glucose oxidase are more substrate-specific than those based on GDH. In GDH- based systems, GDH-FAD and GDH-NAD strips do not cross react with sugars other than glucose, whereas GDH-PQQ is non specific. Maltose, galactose and xylose will be misinterpreted as glucose by GDH-PQQbased sensors $(3,11)$.

U.S Food and Drug Administration (FDA) has listed any product containing or metabolized into maltose, galactose, or xylose, as potential "interfering products" with GDH-PQQ strips. These include, Extraneal (icodextrin) peritoneal dialysis solution; some immunoglobulins including Octagam 5\%, WinRho SDF Liquid, Vaccinia Immune Globulin Intravenous (Human), and HepGamB; Orencia (abatacept); Adept adhesion reduction solution (4\% icodextrin); and BEXXAR radioimmunotherapy agent. According to FDA, Accu-Chek and Free Style are two strips which use GDH-PQQ. FDA advices to avoid using GDH-PQQ glucose test strips in health care facilities and cautions that if they are used "NEVER use them on patients who are receiving interfering products". A possible technical solution to the problem is the use of mutant forms of GDH-PQQ involving amino acid substitution, which have good enzymatic activity for glucose but reduced reactivity for other-sugars $(3,11)$.

The majority of patients as well as many health care providers are unaware of the magnitude of the potential inaccuracy of glucometer results. None of these errors is reason enough for advising against the use of this technology, but we need to educate patients and health care providers about these limitations.

\section{Summary}

Capillary whole blood glucose monitoring has considerably improved the management of diabetes. Nevertheless, there are situations where finger stick glucose measurements are not reliable. Physicians and health care personnel should be aware of "Pseudohypoglycemia" and "Pseudohyperglycemia" where the capillary blood sugars do not correlate with venous plasma glucose. Caution must be exercised in accepting the results as equivalent or using as substitutes for a laboratory blood glucose result. Clinicians should always correlate the blood sugar readings with the clinical findings in taking their management decisions. 


\section{References}

1. Boyd R, Leigh B, Stuart P. Capillary versus venous bedside blood glucose estimations. Emerg Med J 2005; 22: 177-9.

2. Schrot RJ, Patel KT, Foulis P. Potential variables altering home glucose measurement. Evaluation of inaccuracies in the measurement of glycemia in the laboratory, by glucose meters, and through measurement of hemoglobin A1c. Clinical Diabetes 2007; 25: 243-49.

3. Clarke L, Cox D, Goder-Frederick L, Carter W, Pohl S. Evaluating clinical accuracy of systems for self-monitoring of blood glucose. Diabetes Care 1987; 10: 622-8.

4. Olansky L, Kennedy L. Finger-stick glucose monitoring. Issues of accuracy and specificity. Diabetes Care 2010; 33(4): 748-9.

5. Nichols J. What is accuracy and how close must agreement be? Diabetes Technol Therapeut 2005; 7: 558 -62.

6. Khoury ME, Yousuf F, Martin V, Cohen RM. Pseudohypoglycemia: A cause for unreliable finger- stick glucose measurements. Endocrine Practice 2008; 14(3): 337-9.

7. Sacks DB, Bruns DE, Goldstein DE, Maclaren NK, McDonald JM, Parrott M. Guidelines and recom- mendations for laboratory analysis in the diagnosis and management of diabetes mellitus. Clinical Chemistry 2002; 48: 436-72.

8. Diabetes Supply and Resources. Reviews of diabetic testing supplies, diabetes monitoring, glucose tester, blood glucose monitors, insulin pump, insulin syringe, lancet, etc. www.diabetessupplyresources.com (accessed date 27th May 2011).

9. Hirose T, Mita T, Fujitani Y, Kawamori R, Wadata H. Glucose monitoring after fruit peeling: Pseudo-hyperglycemia when neglecting hand washing before finger tip blood sampling. Diabetes Care 2011; 34: 596-7.

10. Hortensius J, Slingerland RJ, Kleefstra N, Logtenberg SJJ , Klaas, Groenier KH, Houweling ST, Bilo HJG. Selfmonitoring of blood glucose: The use of the first or the second drop of blood. Diabetes Care 2011; 34: 556-60.

11. Frias JP, Ellison JM, Lim CG, Montandon CM. Review of adverse events associated with false glucose readings measured by GDH-PQQ-based glucose test strips in the presence of interfering sugars. Diabetes Care 2010; 33(4): $728-9$. 